\title{
Histone deacetylase inhibition reverses sepsis-induced susceptibility to Pseudomonas aeruginosa pneumonia
}

Fanny Alby-Laurent, 1,2Julie Toubiana, 1,2 Marine Torta², Christophe Rousseau2, Laurence Romy², Jean-François Llitjos 2,3 Jean-Paul Mira ${ }^{1,3}$, Frédéric Pène, ${ }^{1,3}$ \& Jean-Daniel Chiche1,3

Dpt. of Pediatrics, Hôpital Necker-Enfants malades; ${ }^{2}$ Cochin Institute, INSERM U1016; ${ }^{3}$ ntensive care Unit, Hôpital Cochin, Université Paris-Descartes, Paris France

\section{Introduction}

- Sepsis induces long lasting alterations of transcriptional programs, subsequent protracted inflammation, sepsis-induced immune suppression (SIIS), secondary infections, and death (1-3)

- A shift toward repressive histone modifications with an overexpression of histone desacetylases (HDACs) is observed in patients with SIIS (4-5)

- We hypothesized that HDACs inhibitors could prevent sepsis-induced overexpression of HDACs, repressive gene expression and thus prevent SIIS.

- To test this hypothesis, we studied the effects of histone deacetylases (HDAC) inhibition with Trichostatin A (TSA) in a murine model of SIIS and secondary pneumonia

\section{Material and methods}

- C57BL/6 mice were treated with TSA $(2 \mathrm{mg} / \mathrm{kg}$ intraperitoneal) or saline serum (CTL) $30 \mathrm{~min}$ before induction of sepsis by cecal ligation puncture (CLP). Sham mice did not undergo CLP. - Surviving mice underwent intratracheal instillation of $P$. aeruginosa $\left(2-2.5 \times 10^{6} \mathrm{CFU}\right) 8$ days after CLP

- We evaluated the effect of TSA on histone 3 acetylation in T-cells by Luminex Histone PTM Multiplex

- Effect of TSA on SIII was evaluated by: - Pneumonia survival, through Kaplan-Meier curves and compared by the log rank test

- Bacterial clearance in BAL (CFU) at 4h of infection

- Lymphocytes sepsis-induced abnormalities 1 and 8 days after initial CLP by flow cytometry (apoptosis, PD-1 and PDL-lexpression)

- Continuous variables were represented as means \pm SD and compared using Student T-test $P<0.05$, represented as * and ${ }^{* *}$ indicated statistically significant differences

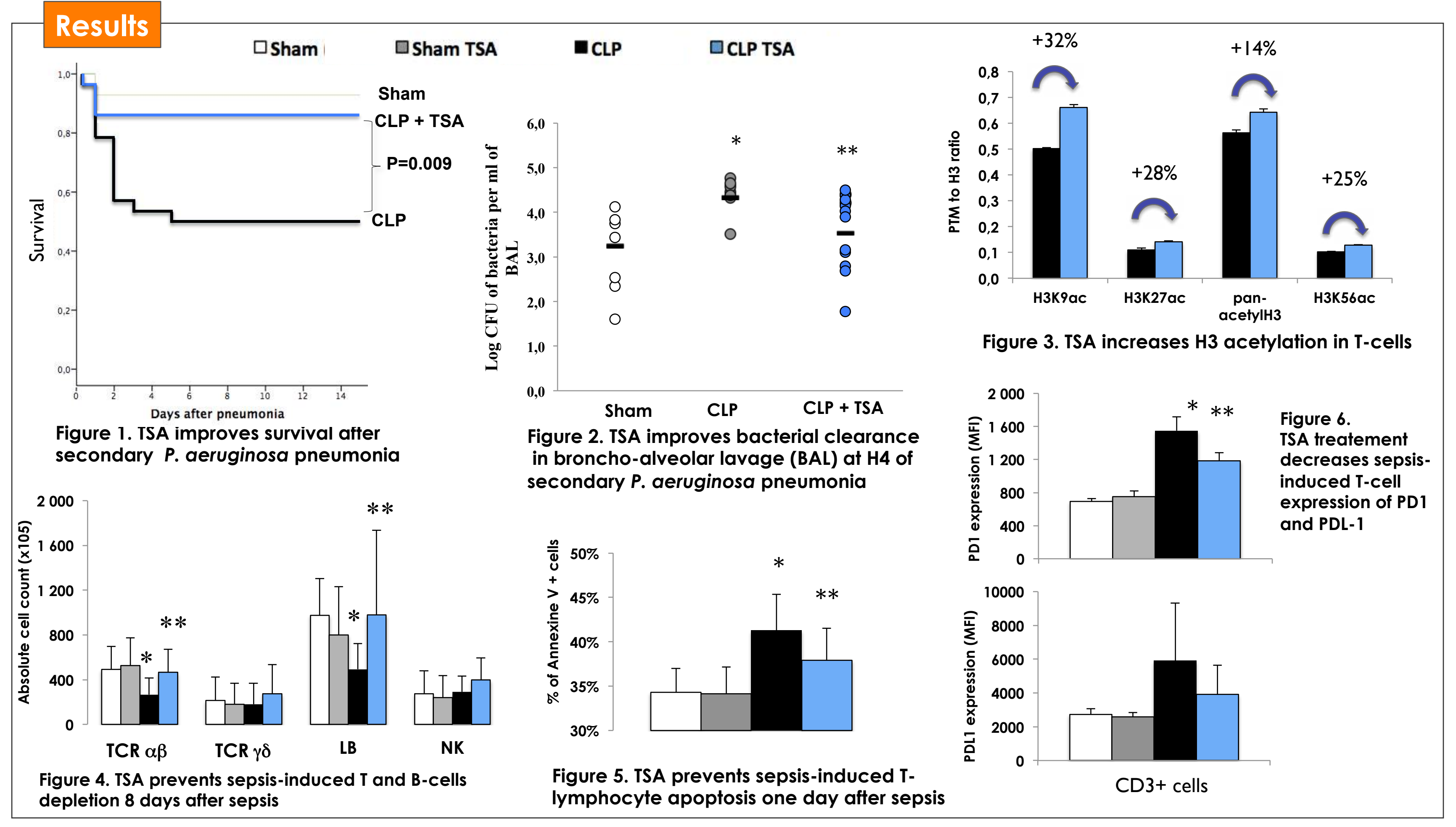

\section{Conclusion}

- HDAC inhibition by TSA improves survival and bacterial clearance in our murine model of secondary P.aeruginosa pneumonia

TSA has an impact on H3 acetylation and T-cell phenotype and prevents from sepsis-induced T-cell apoptosis and PD-1/PDL-1 overexpression

- These results confirm that sepsis-induced epigenetic changes contribute to the advent of SIIS. 